\title{
Factors determining operative reduction in intussusception in children aged less than fourteen years ${ }^{*}$
}

\author{
Tariq O. Abbas ${ }^{\#}$, Noora AlShahwani, Gaby Jabbour, Mansour Ali
}

Department of Pediatric Surgery, Hamad General Hospital, Doha, Qatar

Email: "tariq2c@hotmail.com

Received 20 August 2013; revised 17 September 2013; accepted 23 September 2013

Copyright (C) 2013 Tariq O. Abbas et al. This is an open access article distributed under the Creative Commons Attribution License, which permits unrestricted use, distribution, and reproduction in any medium, provided the original work is properly cited.

\begin{abstract}
Aim: To delineate the factors associated with operative reduction of intussusception, in contrast to enema reduction only without surgical intervention, in children aged less than 14 years. Methods: We retrospectively reviewed the records of all children aged $<14$ years who experienced intussusception between 2002 and 2012 and were treated at a single hospital in Qatar. Clinical outcomes were correlated with age, sex, clinical symptoms, diagnostic- and treatmentrelated characteristics, and length of hospital stay. Results: A total of 141 children were diagnosed and treated for intussusception over 11 years. The male to female ratio of intussusception was 1.4:1. The length of hospital stay was longer in patients who underwent operative reduction than those that did not. Conclusion: Delay in presentation did not decrease the success of radiologic reduction and did not increase the risk of operative intervention and bowel resection.
\end{abstract}

Keywords: Operative Reduction; Intussusceptions; Children

\section{INTRODUCTION}

Intussusception is a common cause of abdominal pain and bowel obstruction in children. Most of these intussusceptions are considered idiopathic in origin as there is usually no identifiable pathology. The underlying cause of intussusception in most infants is unknown, but has been associated with several pathogens, including adenoviruses [1-10].

Although the etiology of intussusceptions remains unclear, adenovirus infection has been associated with an

\footnotetext{
"Authors' Contributions: TA was responsible for most of the proposal writing, data analysis and manuscript writing. NA assisted in data collection and analysis. MA participated in manuscript structuring. ${ }^{\#}$ Corresponding author.
}

increased risk of intussusceptions. Only about $6 \%$ of patients (range, $1.5 \%$ to $12 \%$ ) have intussusceptions, which are associated with a pathologic defect, primarily Meckel's diverticulum [11]. Most episodes of intussusception, termed "idiopathic", arise in the ileum because of lymphoid hyperplasia of Peyer's patches, [12], suggesting a response to infection. An infective etiology is further suggested by the presence, in about $50 \%$ of children with intussusception, of viral shedding in the stools, together with the demonstration of virus particles in pathologic specimens [13].

Although intussusception remains the most common cause of acute intestinal obstruction in infants and young children, it was found to be a side-effect of a vaccine administered to infants in the USA in 1998 for the prevention of rotavirus gastroenteritis. This resulted in a global need to determine the incidence of intussusception before the introduction of new rotavirus vaccines [14].

Children with intussusceptions in developed countries are likely to experience a favorable clinical outcome because of timely diagnosis and early treatment with the less invasive procedure of enema reduction. By contrast, in developing countries, delayed diagnosis with ensuing bowel necrosis, followed by attempted operative reduction, has been associated with high fatality rates, e.g., $18 \%$ in Nigeria, $20 \%$ in Indonesia and up to $54 \%$ in Ethiopia [15].

The treatment of intussusception has evolved from primarily operative management to radiologic reduction with either air or barium contrast. Radiologic reductions of intussusception have been found to decrease the length of hospitalization, shorten recovery time, and reduce the risk of complications associated with major abdominal surgery. The average success rate of radiologic reduction is $80 \%$, but it varies widely [16].

This retrospective analysis evaluated the factors associated with operative reduction of intussusceptions, in contrast to enema reduction alone without the need for surgical intervention, in children aged $<14$ years. 


\section{METHODS}

The medical records of all children aged $<14$ years hospitalized at Hamad General Hospital, Doha, Qatar, from January 2002 through December 2012 were reviewed. Patients who met the definition for intussusception, as determined by the Brighton Collaboration, were identified using the ICD-9 diagnosis code 560.0. Patients were included if the diagnosis of intussusception was verified by imaging (ultrasound, enema, and/or computed tomography $[\mathrm{CT}]$ ) or surgical exploration. Patients were excluded if their records could not validate the diagnosis of intussusceptions.

For each identified child, we extracted information on demographics, admission and discharge dates, clinical signs and symptoms and their duration, and diagnostic and treatment procedures. Data were summarized on standardized questionnaires entered into an electronic database and checked for accuracy; data extraction and entry were performed by the same investigator.

Clinical outcomes were analyzed relative to patient age, sex, clinical signs, date of hospitalization and diagnostic and treatment related characteristics. Because early diagnosis and treatment could provide better outcomes, we compared the length of hospital stay of patients admitted $<24$ hours and $\geq 24$ hours after the onset of symptoms.

Incidence rates were calculated by using census data, and ratios with $95 \%$ confidence intervals were calculated using Poisson regression data.

All radiologic reduction techniques were performed under fluoroscopic guidance using liquids, with the number of reduction attempts at the discretion of individual radiologists. Patients were usually in the prone position, although the supine and oblique positions were also utilized. Conscious sedation was not utilized. A rectal catheter was inserted into each patient, and the buttocks were taped to prevent contrast leakage. Liquid reduction was attempted using either barium or water-soluble diatrizoate meglumine (Gastrografin), prepared by routine methods. A column of contrast was established 1 meter above the table. Typically, 3 consecutive attempts of 3 minutes each were performed.

Descriptive statistics were used to summarize the demographic characteristics of patients. Results are reported as mean \pm standard deviation or medians and inter-quartile ranges.

\section{RESULTS}

\subsection{Patient Characteristics}

Review of the medical records of our center identified 141 children aged $<14$ years who were diagnosed and treated for intussusceptions over the 11-year study period. These patients included 83 boys and 58 girls (ratio,
1.4:1). Of these 141 patients, 47 (31.7\%), of mean age 13.6 months, underwent operative management and 96 (68.3\%), of mean age 14.0 months, underwent enema reduction.

\subsection{Delay in Presentation and Symptoms}

Delay in presentation did not decrease the success of radiologic reduction. The rate of operative intervention was lower in patients who presented $<24$ hours than in those who presented $\geq 24$ hours after symptom onset ( $22.2 \%$ vs. $41.5 \%$; Table 1$)$.

Symptoms were similar in patients managed with enema and operative reduction (Table 2).

\subsection{Length of Stay}

Length of stay ranged from 1 to 7 days (mean, 2.6 days). Length of stay was longer in patients who underwent operative than enema reduction, both in patients who presented $<24$ hours and $\geq 24$ hours after symptom onset (Table 3).

\subsection{Coexisting Conditions}

Generally speaking, the number of coexisting conditions was greater in patients managed with enema reduction than in those who needed surgical intervention (Table 4).

Table 1. Duration of symptoms in patients managed surgically or with enema reduction.

\begin{tabular}{ccc}
\hline Enema reduction & Surgical reduction & $\begin{array}{c}\text { Average symptom } \\
\text { duration (days) }\end{array}$ \\
\hline 46 & 16 & $\leq 1$ \\
9 & 6 & 2 \\
4 & 2 & 3 \\
3 & 1 & 4 \\
2 & 0 & 5 \\
1 & 0 & $>7$ days \\
4 & 1 & unknown \\
\hline
\end{tabular}

Table 2. Symptoms in patients managed surgically and by enema reduction.

\begin{tabular}{ccc}
\hline Enema reduction & Surgical reduction & Symptoms \\
\hline 8 & 11 & Bile stained vomiting \\
8 & 8 & Nonbilious vomiting \\
15 & 10 & Abdominal pain \\
12 & 15 & Bleeding per rectum \\
3 & 1 & Fever \\
\hline
\end{tabular}


Table 3. Lengths of stay in patients managed with enema reduction and operatively.

\begin{tabular}{cccc}
\hline Total & Operative & Non operative & \\
\hline $2.15 \pm 1.34$ & $3.73 \pm 1.26$ & $1.57 \pm 0.88$ & Less than $24 \mathrm{~h}$ \\
$2.07 \pm 1.16$ & $2.85 \pm 1.46$ & $1.79 \pm 0.83$ & More than 24 hours \\
\hline
\end{tabular}

Table 4. Coexisting conditions in patients managed surgically and with enema reduction.

\begin{tabular}{ccc}
\hline Enema reduction & Surgical reduction & Coexisting conditions \\
\hline 1 & None & UDT \\
1 & None & Hernia \\
1 & None & G6PD \\
1 & None & CHD \\
1 & None & Malrotation \\
\hline
\end{tabular}

\subsection{Presence of Free Peritoneal Fluid on Ultrasound}

Peritoneal fluid was present in 10 patients $(9 \%)$ who required only enema reduction and in $7(16 \%)$ who required surgical intervention.

\subsection{Recurrent Intussusceptions}

Three patients experienced recurrences, with all three managed with enema reduction only.

\section{DISCUSSION}

Intussusception is a common childhood problem worldwide, with high rates of morbidity and mortality. In developing nations, the mortality may be as high as $20 \%$. [11]

Nonoperative reduction of intussusceptions, performed by a radiologist using hydrostatic or pneumatic pressure under fluoroscopic or sonographic guidance, is usually successful. Nonoperative reduction has been shown to reduce length of hospital stay, shorten recovery time, decrease hospital costs, and decrease the risk of complications related to abdominal surgery [17]. The success rate of enema reduction has been found to vary widely, usually averaging $75 \%[18,19]$. In our series, however, the success rate was somewhat lower, $68.7 \%$.

Studies have reported that the median ages of children requiring operative and non-operative reduction were similar $(0.7$ years $[0.4,2.5$ years $]$ vs 0.9 years $[0.5,2.0$ years]), although children aged 1 to 2 years were less likely to require operative reduction [11]. We found that children who underwent operative reduction were less likely to have a coexisting condition associated with intussusceptions, in contrast to previous findings.

Operative reduction has been associated with a longer length of hospital stay, especially when bowel resection was performed. This results in higher hospital costs, as well as inefficient use of health care resources. In addition, the need for laparotomy increases morbidity rates, pain, and long-term risks of adhesive bowel obstruction. Rarely, a delay in diagnosis may lead to bowel necrosis and overwhelming sepsis, a potentially lethal condition [19]. Studies have shown that $6 \%$ to $53 \%$ of children require surgical reduction, including children who fail enema reduction and those with contraindications to enema reduction [13,20-23].

Although intussusception is a common surgical condition in children, there are no current guidelines in Qatar regarding the treatment of children with this condition. Duration of symptoms did not affect patient outcomes, with the percentage of patients having symptoms $<24$ hours being lower in patients requiring surgical intervention.

Over the last two years, however, attempts have been made to standardize the referral system for children with intussusception. Patients suspected of having this condition are sent to the Pediatric Emergency Centre (PEC) from different regions throughout the country for ultrasound examination. Moreover, these patients are immediately evaluated by the on-call pediatric surgeon, with patients confirmed to have intussusceptions by ultrasound admitted to the hospital. Hydrostatic reduction is scheduled as soon as possible, to be performed by the same radiologist who performed the ultrasound. Nevertheless, it is impractical to refer all children with abdominal pain and vomiting to the radiology department of the main hospital in the country and to initiate this protocol. Thus, our results should stimulate efforts toward educating community-based physicians about the diagnosis and management of intussusception.

Although the presence of free peritoneal fluid on ultrasound was regarded as prognostic of a higher rate of failure of enema reduction, we found that free peritoneal fluid was present in $16 \%$ of patients requiring surgical intervention, compared with only $9 \%$ of patients who underwent successful enema reduction. Similarly, recurrent intussusception was not an indicator for surgical intervention.

This study had several limitations, including its retrospective design. Moreover, it was difficult to obtain accurate birth-cohort data for the catchment population. Nevertheless, the strength of this retrospective study was its analysis of patients treated at the only tertiary care facility in the country, with complete evaluations and reliable documentation.

\section{CONCLUSION}

In conclusion, this report, describing our 11-year experience of intussusceptions in Qatar, provides accurate baseline data. We found that a delay in presentation did 
not decrease the success rate of radiologic reduction and did not increase the risk of operative intervention and bowel resection.

\section{REFERENCES}

[1] Clarke Jr., E.J., Phillips, I.A. and Alexander, E.R. (1969) Adenovirus infection in intussusception in children in Taiwan. JAMA, 208, 1671-1674. doi:10.1001/jama.1969.03160090031007

[2] Nicolas, J.C., Ingrand, D., Fortier, B., et al. (1982) A one-year virological survey of acute intussusception in childhood. Journal of Medical Virology, 9, 267-271. doi:10.1002/jmv.1890090404

[3] Montgomery, E.A. and Popek, E.J. (1994) Intussusception, adenovirus, and children: A brief reaffirmation. Human Pathology, 25, 169-174. doi:10.1016/0046-8177(94)90274-7

[4] Hsu, H.Y., Kao, C.L., Huang, L.M., et al. (1998) Viral etiology of intussusception in Taiwanese childhood. The Pediatric Infectious Disease Journal, 17, 893-898. doi:10.1097/00006454-199810000-00009

[5] Bode, C.O. and Omilabu, S.A. (2002) Viral isolates of intussusception in Nigerian infants. South African Journal of Surgery, 40, 57-58.

[6] Guarner, J., de Leon-Bojorge, B., Lopez-Corella, E., et al. (2003) Intestinal intussusception associated with adenovirus infection in Mexican children. American Journal of Clinical Pathology, 120, 845-850. doi:10.1309/LBRNGF9MJW2MHT97

[7] Selvaraj, G., Kirkwood, C., Bines, J., et al. (2006) Molecular epidemiology of adenovirus isolates from patients diagnosed with intussusception in Melbourne, Australia. Journal of Clinical Microbiology, 44, 3371-3373. doi:10.1128/JCM.01289-06

[8] Daneman, A. and Navarro, O. (2003) Intussusception part 1: A review of diagnostic approaches. Pediatric Radiology, 33, 79-85.

[9] Robinson, C.G., Hernanz-Schulman, M., Zhu, Y., et al. (2004) Evaluation of anatomic changes in young children with natural rotavirus infection: Is intussusception biologically plausible? The Journal of Infectious Diseases, 189, 1382-1387. doi:10.1086/382655

[10] Parasher, V.D., Holman, R.C. and Cummings, K.C. (2000) Trends in intussusceptions associated hospitalizations and deaths among US Infants. Pediatrics, 106, 1413-1421. doi:10.1542/peds.106.6.1413

[11] Boudville, I.C., Phua, K.B., Quak, S.H., et al. (2006) The epidemiology of paediatric intussusception in Singapore: 1997 to 2004. ANNALS Academy of Medicine Singapore, 35, 674-679.

[12] Jen, H.C. and Shew, S.B. (2009) The impact of hospital type and experience on the operative utilization in pediatric intussusception: A nationwide study. Journal of Pediatric Surgery, 44, 241-246. doi:10.1016/j.jpedsurg.2008.10.050

[13] Bratton, S.L., Haberkern, C.M., Waldhausen, J.H.T., et al. (2001) Intussusception: Hospital size and risk of surgery. Pediatrics, 107, 299-303. doi:10.1542/peds.107.2.299

[14] Nakagomi, T., Takahashi, Y., Arisawa, K., et al. (2006) A high incidence of intussusception in Japan as studied in a sentinel hospital over a 25-year period (1978-2002). Epidemiology \& Infection, 134, 57-61. doi:10.1017/S0950268805004644

[15] Bhowmick, K., Kang, G., Bose, A., et al. (2009) Retrospective surveillance for intussusception in children aged less than five years in a South Indian tertiary-care hospital. Journal of Health, Population and Nutrition, 27, 660665.

[16] Murphy, T.V., Gargiullo, P.M., Massoudi, M.S., et al. (2001) Intussusception among infants given an oral rotavirus vaccine. The New England Journal of Medicine, 344, 564-572. doi:10.1056/NEJM200102223440804

[17] Samad, L., Marven, S., El Bashir, H., et al. (2012) Prospective surveillance study of the management of intussusception in UK and Irish infants. British Journal of Surgery, 99, 411-415. doi:10.1002/bjs.7821

[18] Tate, J.E., Simonsen, L., Viboud, C., et al. (2008) Trends in intussusception hospitalizations among US infants, 1993-2004: Implications for monitoring the safety of the new rotavirus vaccination program. Pediatrics, 121, Article ID: e1125.

[19] Somme, S., To, T. and Langer, J.C. (2006) Factors determining the need for operative reduction in children with intussusception: A population-based study. Journal of Pediatric Surgery, 41, 1014-1019. doi:10.1016/j.jpedsurg.2005.12.047

[20] Boudville, I.C., Phua, K.B., Quak, S.H., et al. (2006) The epidemiology of paediatric intussusception in Singapore: 1997 to 2004. ANNALS Academy of Medicine Singapore, 35, 674-679.

[21] Le Masne, A., Lortat-Jacob, S. and Sayegh, N. (1999) Intussusception in infants and children: Feasibility of ambulatory management. European Journal of Pediatrics, 158, 707-710.

[22] Daneman, A. and Navarro, O. (2004) Intussusception part 2: An update on the evolution of management. Pediatric Radiology, 34, 97-108.

[23] Applegate, K.E. (2006) Intussusception in children: Diagnostic imaging and treatment. In: Blackmore, C.C. and Medina, S., Eds., Evidence-Based Imaging, Springer, New York, 475-492. 